\title{
INCIDENCE COLORING-COLD CASES
}

\author{
FRANTIŠEK KARdošn ${ }^{1}$, MÁRIA MACEKOVÁ ${ }^{2}$ \\ Martina MockovČIAKOvÁ ${ }^{3}$, Éric Sopena ${ }^{1}$ \\ AND \\ Roman SOTÁK ${ }^{2}$ \\ ${ }^{1}$ Univ. Bordeaux, Bordeaux INP, CNRS, LaBRI, \\ UMR5800, F-33400 Talence, France \\ ${ }^{2}$ Institute of Mathematics \\ P.J. S̆afárik University in Košice \\ Jesenná 5, 04001 Košice, Slovakia \\ ${ }^{3}$ European Centre of Excellence NTIS \\ University of West Bohemia \\ Pilsen, Czech Republic \\ e-mail: frantisek.kardos@labri.fr \\ maria.macekova@upjs.sk \\ mmockov@ntis.zcu.cz \\ eric.sopena@labri.fr \\ roman.sotak@upjs.sk
}

\begin{abstract}
An incidence in a graph $G$ is a pair $(v, e)$ where $v$ is a vertex of $G$ and $e$ is an edge of $G$ incident to $v$. Two incidences $(v, e)$ and $(u, f)$ are adjacent if at least one of the following holds: (i) $v=u$, (ii) $e=f$, or (iii) edge $v u$ is from the set $\{e, f\}$. An incidence coloring of $G$ is a coloring of its incidences assigning distinct colors to adjacent incidences. The minimum number of colors needed for incidence coloring of a graph is called the incidence chromatic number.

It was proved that at most $\Delta(G)+5$ colors are enough for an incidence coloring of any planar graph $G$ except for $\Delta(G)=6$, in which case at most 12 colors are needed. It is also known that every planar graph $G$ with girth at least 6 and $\Delta(G) \geq 5$ has incidence chromatic number at most $\Delta(G)+2$.

In this paper we present some results on graphs regarding their maximum degree and maximum average degree. We improve the bound for planar graphs with $\Delta(G)=6$. We show that the incidence chromatic number is at
\end{abstract}


most $\Delta(G)+2$ for any graph $G$ with $\operatorname{mad}(G)<3$ and $\Delta(G)=4$, and for any graph with $\operatorname{mad}(G)<\frac{10}{3}$ and $\Delta(G) \geq 8$.

Keywords: incidence coloring, incidence chromatic number, planar graph, maximum average degree.

2010 Mathematics Subject Classification: 05C15. 


\section{REFERENCES}

[1] M. Bonamy, B. Lévêque and A. Pinlou, 2-distance coloring of sparse graphs, J. Graph Theory 77 (2014) 190-218.

doi:10.1002/jgt.21782

[2] R.A. Brualdi and J.J. Quinn Massey, Incidence and strong edge colorings of graphs, Discrete Math. 122 (1993) 51-58. doi:10.1016/0012-365X(93)90286-3

[3] B. Guiduli, On incidence coloring and star arboricity of graphs, Discrete Math. 163 (1997) 275-278. doi:10.1016/0012-365X(95)00342-T

[4] S.L. Hakimi, J. Mitchem and E. Schmeichel, Star arboricity of graphs, Discrete Math. 149 (1996) 93-98.

doi:10.1016/0012-365X(94)00313-8

[5] M. Hosseini Dolama, É. Sopena and X. Zhu, Incidence coloring of $k$-degenerated graphs, Discrete Math. 283 (2004) 121-128. doi:10.1016/j.disc.2004.01.015

[6] M. Hosseini Dolama and É. Sopena, On the maximum average degree and the incidence chromatic number of a graph, Discrete Math. Theor. Comput. Sci. 7 (2005) 203-216.

[7] D.P. Sanders and Y. Zhao, Planar graphs of maximum degree seven are class I, J. Combin. Theory Ser. B 83 (2001) 201-212.

doi:10.1006/jctb.2001.2047

[8] D. Yang, Fractional incidence coloring and star arboricity of graphs, Ars Combin. 105 (2012) 213-224.

Received 9 October 2017

Revised 25 April 2018

Accepted 26 April 2018 